\title{
S29. Improvement of compliance - quality assurance - increased quality of life in community care for schizophrenics (supported by an educational grant from Lundbeck DK)
}

THE NEED FOR QUALITY ASSURANCE FOR A BETTER QUALITY OF LIFE IN CHRONIC SCHIZOPHRENIA

\section{SJ Dencker}

Department of Clinical Neuroscience, Section of Psychiatry, Lillhagens Hospital, Box 3005, S-422 03 Hisings Backa, Sweden

The therapy-rehabilitation programmes for chronic schizophrenia have reduced the need for custody care considerably. However, the patients in a community care setting are usually characterized by an unstable social situation, a poor quality of life and relapse leading to hospital admission.

Rest psychotic symptoms and psychotic relapse have been focused upon as risk factors in community care. Eventually, the interest for a more professional care in the long-term perspective has substantially increased. Such aspects have highlighted compliance as an essential factor for acceptance of the continuation therapy programme for the chronic schizophrenic, his relatives included.

There are thus distinct needs for a better compliance in the long-term community care of the chronic schizophrenic. Quality assurance and medical audit aspects must be used for the monitoring of the process, based on good clinical psychiatric paractice. Thus a better quality of life for the chronic schizophrenic will eventually be achieved. The aspects and results of the symposium will be published in a consensus paper.

\section{THE CONTEXT AND CONTENT OF COMPLIANCE.}

P.E. Bebbington M.A. Ph.D. FRCP. FRCPsych Honorary Consultant Psychiatrist and Hon Reader in Social and Epidemiological Psychiatry

MRC Social and Community Psychiatry Unit, Institute of Psychiatry, London SE5 8QU

The concept of compliance is inherently linked with the nature of the practice of medicine and the resulting relationship between physician and patient. The medical tradition is based on the idea that the essential product, treatment, is defined by the vendor, as expert. The patient is thus supposed to submit to a directive from the physician. This model is unlikely ever to have more than provisional legitimacy, and it falls down when patients and physicians have different views about the disease, the treatment and their own interaction. Without proper acknowledgement of the social realities of the doctor-patient relationship, compliance will always be tentative. Where treatments are genuinely effective the cost in terms of lost health product will be considerable. Improvement in compliance is dependent on the individual physician being able to appreciate the social context within which treatments are offered and the significance of the patient's beliefs, both general and specific, about illness and treatment. In this paper I interpret the issue of compliance in terms of the sociology of illness and the 'health belief model', and use this to provide guidelines for increasing the effectiveness of treatment. 


\section{BASIC ELEMENTS IN BIOBEHAVIORAL TREATMENT \& REHABILITATION OF SCHIZOPHRENIA}

RP Liberman

Clinical Research Center for Schizophrenia, UCLA

Department of Psychiatry \& Biobehavioral Sciences, West

LosAngeles VA Medical Center, Wilshire \& Sawtelle Blvds., LosAngeles, CA 90073, USA

The pervasive and disabling cognitive impairments and symptoms of schizophrenia require a comprehensive. coordinated, continuous, and integrated biobehavioral treatment and rehabilitation program. The elements of this program include accurate diagnosis coupled with judicious types and doses of antipsychotic medication; ongoing, quantitative measurement of psychopathology keyed to adjustments in psychoactive medication that optimizes the benefit/risk ratio; functional assessment which engages the patient and family (or other natural caregivers) as informed consumers of services who are actively involved in treatment planning and implementation; training in social and independent living skills; behavioral family management; supported employment and housing; and social security benefits, basic medical and dental care.

These elements must be delivered by a team that offers 24 hours per day and 7 days per week accessibility and responsiveness. An important element in a responsive and effective community support program is the case manager or case management team that provides assertive outreach for evaluation and treatment in the home and other community-based locales. Providing flexible levels of intervention, linked to the changing needs of the patient, is extremely important in the successful individualizing of services. When these basic elements can be provided early in the course of schizophrenia, then recovery from schizophrenia is possible.

\section{PSYCHOEDUCATION AND RELAPSE PREVENTION}

\section{MJ Goldstein}

Departments of Psychology and Psychiatry, University of California, Los Angeles, 405 Hilgard, Los Angeles, California, 90024-1563, USA

The trend towards briefer inpatient stays for psychotic patients has led to a greater emphasis on community care. It has also brought the relatives of patients into the therapeutic process, as caretakers and support providers for patients. Psychoeducational programs have been developed to bridge the gap between treatment personnel and patients and their relatives. They are also important adjuncts to encouraging compliance with maintenance medication.

The various psychoeducational programs used in controlled clinical trials have been designed to assist relatives and patients to: (1) integrate the most recent psychotic experience, (2) accept vulnerability to future episodes, (3) understand the need for continued reliance on medication, (4) appreciate the role of stressful life events on triggering relapse, and (5) learning to distinguish variations in normal personality from incipient signs of the disorder.

Five controlled trials with schizophrenic patients have revealed that the addition of a psychoeducational family program to regular maintenance neuroleptic treatment can reduce relapse in the short term by one third. Variations in the format of the psychoeducational programs tested still leave unanswered questions as to the "active ingredients" within them. It is unclear whether these programs should be targeted at relatives only, or whether the critical ingredient is involvement of relatives and patients in such a program, or whether specific therapeutic modalities are required for relapse prevention.

Current efforts by our group to expand the psychoeducational model to bipolar patients and their relatives will be described.

\section{NEW DIAGNOSTIC VISTAS}

R FOG

St. Hans Hospital, Psychiatric dept. P, DK-4000 Roskilde, Denmark.

There are many difficulties in establishing criteria for better drug compliance and improved quality assurance in the treatment of schizophrenic patients.

The most serious problem (especially with first episodes) is that of diagnosis. Since no biological markers for schizophrenia have been found the clinician is referred to "symptom collecting" (including social data etc.). In spite of many attempts there is still no general agreement of the concept of schizophrenia. This deplorable fact is also reflected in the everchanging subdivisions of the schizophrenias over the decades: From acute and chronic dementia praecox via simple, hebephrenic, catatonic, paranoid forms, on to positive/negative manifestations, type l/type II forms etc.

The many different rating scales are reflections of the same problem and so are the different diagnostic systems (e.g. DSM and ICD). The recent attempts to make a synthesis of these systems seem promising but raise new problems concerning for example etiological views of psychiatric disorders. 